\title{
Failure analysis of overloaded coulometric hydrogen sensor
}

\author{
Andreas Graff ${ }^{1}$, Wolfram Münchgesang ${ }^{1,2}$, Frank Altmann ${ }^{1}$, Cameliu Himcinschi ${ }^{2}$, Thomas \\ Köhler ${ }^{2}$, Pramit Sood ${ }^{3}$, Jens Zosel ${ }^{3}$ and Michael Mertig ${ }^{3}$ \\ ${ }^{1}$ Fraunhofer Institute for Microstructure of Materials and Systems (IMWS), 06120 Halle, Germany \\ ${ }^{2}$ Technische Universität Bergakademie Freiberg, 09599 Freiberg, Germany \\ ${ }^{3}$ Kurt-Schwabe-Institut für Mess- und Sensortechnik Meinsberg e.V., Kurt-Schwabe-Straße 4, \\ 04736 Waldheim, Germany
}

\section{Summary:}

An irreversible degradation of a coulometric hydrogen sensor is observed after operation at high electrical load. Microstructural investigations were performed on the YSZ ceramic tube of the sensor with outer and inner electrodes to find the root causes for the degradation. Local color changes (blackening) were observed caused by the reduction of the YSZ by oxygen diffusion at grain boundaries under chemical and electrical potential. Crack formation and phase transitions in the YSZ ceramic from cubic to monoclinic can explain the irreversible degradation of the solid electrolyte sensor.

Keywords: gas chromatography, coulometry, hydrogen sensor; Yttria-stabilized zirconia (YSZ)

\section{Background, Motivation and Objective}

Hydrogen is a convenient candidate to store energy produced by surplus green electricity. The key component for converting the electrical energy into chemical energy of hydrogen is the electrolyzer which splits water into hydrogen and oxygen. To ensure the safety during the production, storage, transport and usage of hydrogen gas sensors are mandatory. For the electrolyzer, hydrogen sensors must have a sensitivity of 100 vol.-ppm up to a concentration of 4 vol.- $\%$ hydrogen in an oxygen atmosphere or in air at pressures up to 30 bar and temperatures up to $90^{\circ} \mathrm{C}$. Coulometric solid electrolyte sensors for hydrogen provide the required sensitivity and withstand these operation conditions. To suppress the cross sensitivity against other gases the substances can be separated in advance by gas chromatography. Such sensors have to sustain under harsh environment and high electrical fields causing degradation of the sensor materials and limiting the life time. This study focusses on the solid electrolyte YSZ in the coulometric measurement cell and its structural changes during extreme load conditions.

\section{Description of the Methods}

Microstructural investigations are performed on a gas-tight YSZ ceramic tube (Friatec, $8 \mathrm{~mol} \%$ $Y$ ) after electrical overload. The YSZ tube is the central part of a coulometric sensor device (Zirox $\mathrm{GmbH}$ ). The oxygen transport in and out the tube is controlled by two pairs of $\mathrm{Pt}(99.99 \%$ pure) electrodes fixed by YSZ cement to the inner and outer wall of the tube. One pair is used for measurement and the other one as reference.
The electrodes are also used to measure the difference of the chemical potential between inside and outside the tube. The microstructure of the YSZ ceramic was mainly determined by electron microscopy (SEM Zeiss Supra, TEM FEI Titan) combined with diffraction and analytical methods (Trident EBSD-EDX, EDAX). Additional structural information was obtained by XRD and Raman spectroscopy.

\section{Results}

The experiments started with the conditioning of the sensor under high electrical load. Starting with oxygen transport from outside air to a reforming gas ( 50 vol.-ppm $\mathrm{H}_{2}$ in $\mathrm{N}_{2}$ ) inside the tube for $60 \mathrm{~s}$ at $750{ }^{\circ} \mathrm{C}$. The transport was forced by a voltage of $11 \mathrm{~V}$ and the current was limited to $0.6 \mathrm{~A}$. During the conditioning the tube gets damaged by elevated local current densities and developed remarkable oxygen gas diffusion through the tube wall. After conditioning the voltage at the electrodes was reversed automatically to establish a low oxygen partial pressure inside the tube. Due to the unnoticed gas leakage, a high pumping current was applied for more than $60 \mathrm{~min}$. The structural changes caused by this experiment were investigated in detail.

\subsection{Defect localization and blackening}

To localize the positions of the gas leakage and assumed electrical shorts sites have to be found where the color of the YSZ changes from opaque to black. Known as blackening-effect occurring in reduction atmosphere [1]. After removing the electrodes blackening could be observed only at single spots on the tube surface. To localize more positions laser cross sectioning and inside 
illumination have been performed (Fig.3) before the tube was separated by sawing to get cross sections for further investigations. At cross sections blackening could be observed at the inner electrode. At some positions the blackening reaches the outer surface of the tube indicating possible sites of gas leakage and electrical shorts (Fig.1).

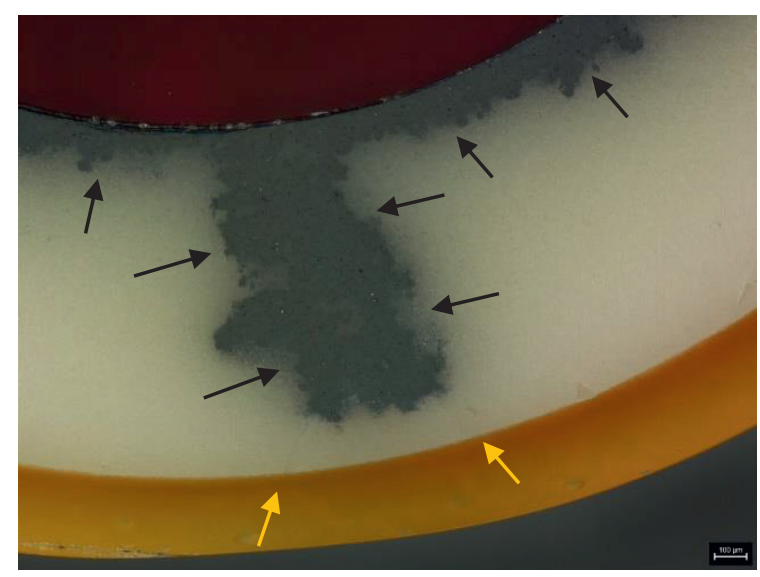

Fig. 1 Light microscope image of mechanical cross section of the YSZ tube. Blackening and cracks are visible in the YSZ ceramic starting at inner surface.

\subsection{Microstructural investigation}

Different analysis methods were applied to correlate the blackening with changes in the microstructure of the YSZ. To determine the grain structure back scatter electron (BSE) imaging and EBSD was applied to identify the different phases of the ceramic. In comparison to unaffected areas void formation and cracks at the grain boundaries could be found in the blackening areas (Fig.2). EBSD analysis reveal the hexagonal Alumina structure of the darker precipitates (Fig.4) seen in the BSE images. Raman measurements indicate the monoclinic Zirconia phase in the blackening areas in contrast to the cubic and tetragonal phases at the reference sites.

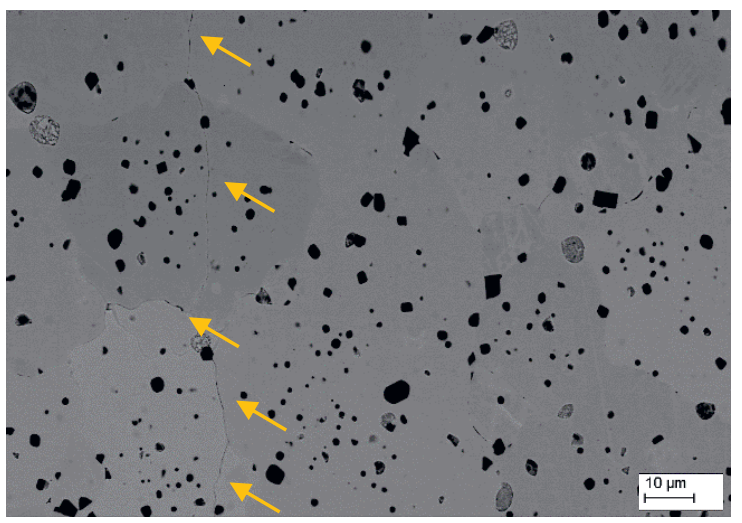

Fig. 2 BSE image of the cross section with grain structure and $\mathrm{Al}_{2} \mathrm{O}_{3}$ inclusions. Micro cracks have formed (left side) in the black regions.

\section{References}

[1] J. Janek.; C. Korte. Electrochemical blackening of yttria-stabilized zirconia - morphological instability of the moving reaction front. Solid State Ionics, 1999, 116, 181-195.

\section{Acknowledgments}

This research was funded by the German Federal Ministry of Education and Research, grant numbers 03ZZ0724A, 03ZZ0724E and 03ZZ0724I within the "HYPOS - Hydrogen Power Storage \& Solutions East Germany" project.

\section{Illustrations, Graphs, and Photographs}
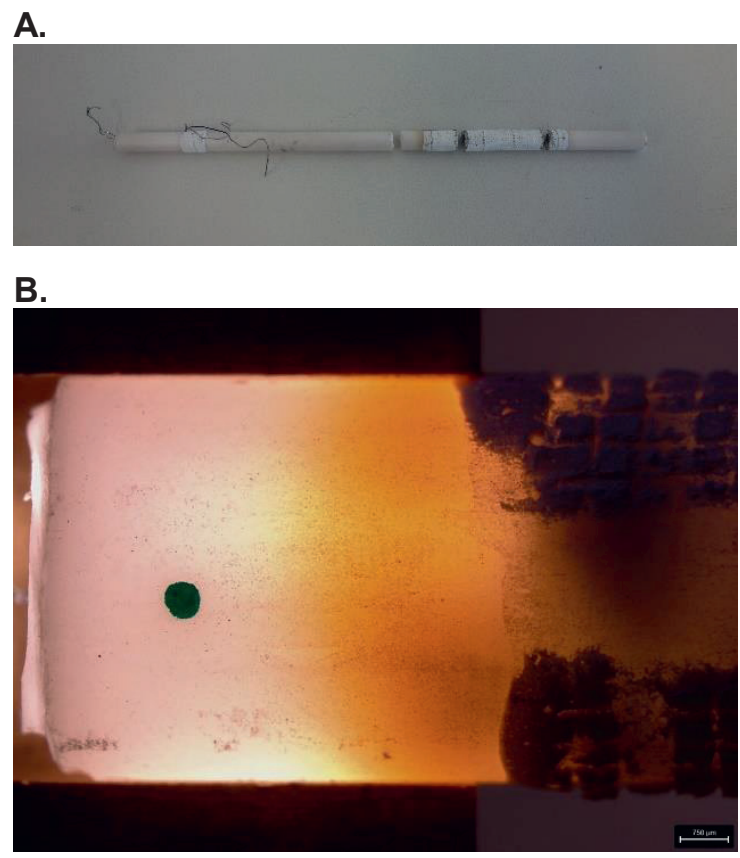

Fig. 3. A. Overview of the sawn YSZ tube with electrodes. B. Part of the ceramic tube with inside illumination at the electrode position. Blackening inside the tube is visible.

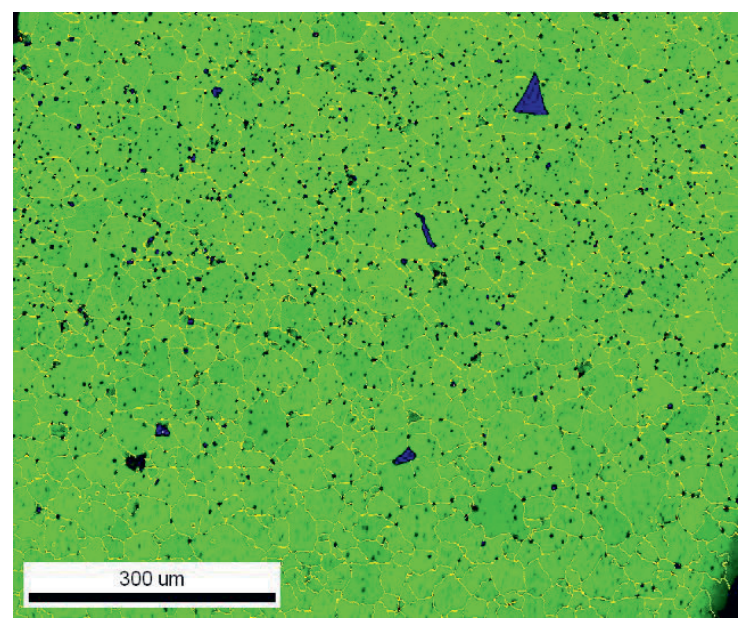

Fig. 4 EBSD phase and quality image of the cross section with grain structure and $\mathrm{Al}_{2} \mathrm{O}_{3}$ (blue) inclusions as well as YSZ (green). 